\title{
Melting of stripe phases and its signature in the single-particle spectral function
}

\author{
Marcin Raczkowski and Fakher F. Assaad \\ Institut für Theoretische Physik und Astrophysik, \\ Universität Würzburg, Am Hubland, D-97074 Würzburg, Germany
}

(Dated: December 6, 2018)

\begin{abstract}
Motivated by the recent experimental data [Phys. Rev. B 79, 100502 (2009)] indicating the existence of a pure stripe charge order over unprecedently wide temperature range in $\mathrm{La}_{1.8-x} \mathrm{Eu}_{0.2} \mathrm{Sr}_{x} \mathrm{CuO}_{4}$, we investigate the temperature-induced melting of the metallic stripe phase. In spite of taking into account local dynamic correlations within a real-space dynamical mean-field theory of the Hubbard model, we observe a mean-field-like melting of the stripe order irrespective of the choice of the next-nearest neighbor hopping. The temperature dependence of the single-particle spectral function shows the stripe induced formation of a flat band around the antinodal points accompanied by the opening a gap in the nodal direction.

PACS numbers: 71.10.Fd, 71.27.+a, 74.72.-h, 79.60.-i
\end{abstract}

In spite of intense theoretical studies over the two last decades, the debate on the microscopic origin of the socalled stripe phases characterized by the combined spin and charge order is far from closed. $\underline{\underline{1}}$ So far, two main scenarios regarding the origin of stripes have been put forward. In the weak-coupling scenario, stripe phases emerge due to nesting properties of the Fermi surface and provide a compromise between the superexchange interaction, which stabilizes the long-range antiferromagnetic (AF) order in the parent Mott insulator, and the kinetic energy of doped holes. In this scenario spin and charge orders occur at the same temperature or charge stripe order sets in only after spin order has developed. An alternative strong-coupling scenario comes from the Coulombfrustrated phase separation suggesting that stripe formation is commonly charge driven $\stackrel{2}{2}$ In this case the onset of charge order appears prior to spin order as the temperature is lowered. However, this scenario does not take into account spin fluctuations which might prevent the spins from ordering at the charge-order temperature $T_{\mathrm{CO}} \stackrel{3}{\underline{3}}$

Similarly, on the experimental side the origin of stripes remained up to now unclear. On the one hand, in the most widely studied stripe-ordered compounds $\mathrm{La}_{1.6-x} \mathrm{Nd}_{0.4} \mathrm{Sr}_{x} \mathrm{CuO}_{4}$ (LNSCO) $)^{\frac{4}{4}}$ and $\mathrm{La}_{2-x} \mathrm{Ba}_{x} \mathrm{CuO}_{4}, \underline{\underline{5}}$ the charge order observed as Bragg maxima at the wave vectors $\boldsymbol{q}_{c}=( \pm 4 \pi \epsilon, 0)$ with $\epsilon \simeq x$ for doping $x \lesssim 1 / 8$ was found to set in at a higher temperature $T_{\mathrm{CO}}$ than the spin order signalized below $T_{\mathrm{SO}}$ by the onset of magnetic Bragg peaks at $\boldsymbol{q}_{s}=\pi(1 \pm 2 \epsilon, 1)$, thus supporting the strong coupling scenario. On the other hand, the interval between $T_{\mathrm{CO}}$ and $T_{\mathrm{SO}}$ was found not to exceed 15 $\mathrm{K}$. In contrast, in the recent resonant soft x-ray studies of the stripe order in $\mathrm{La}_{1.8-x} \mathrm{Eu}_{0.2} \mathrm{Sr}_{x} \mathrm{CuO}_{4}$ (LESCO), $\underline{\underline{6}}$ both temperatures were found to be separated by $35 \mathrm{~K}$. Those findings clearly exclude the nesting scenario of the stripe formation in favor of the electronic origin.

In this paper we address melting of the metallic stripe order and look for a signature of a pure charge-order above $T_{\mathrm{SO}}$. We consider the Hubbard model,

$$
H=-\sum_{i j, \sigma} t_{i j} c_{i \sigma}^{\dagger} c_{j \sigma}+U \sum_{i} n_{i \uparrow} n_{i \downarrow},
$$

with a hopping amplitude $t\left(t^{\prime}\right)$ between the (next-) nearest neighbor sites, respectively, and with $U=10 t$ standing for the on-site Coulomb interaction and solve it within the real-space dynamical mean-field theory (RDMFT). It allows one to handle the leading local part of dynamic correlations exactly. Although the long-range Coulomb interaction might help to stabilize further the stripe order, the great success of the previous RDMFT studies at $T=0$ was a proof that the correct treatment of the on-site interaction alone suffices to stabilize experimentally observed metallic stripes in $\mathrm{LNSCO}^{2} \underline{7}$ Following Ref. 7, we decompose the square lattice into $L_{u}$ stripe supercells with $\mu=1 \ldots L_{c}$ orbitals so that the selfenergy $\boldsymbol{\Sigma}_{\sigma}\left(\boldsymbol{K}, i \omega_{m}\right)$ and noninteracting Green's function $\boldsymbol{G}_{0}\left(\boldsymbol{K}, i \omega_{m}\right)$ become $L_{c} \times L_{c}$ matrices, where the wave vectors $K$ span the folded Brillouin zone (BZ). Furthermore, similarly to the standard DMFT,, the RDMFT approximation neglects the $\boldsymbol{K}$ dependency of the selfenergy while allowing for its spatial dependency, i.e., $\boldsymbol{\Sigma}_{\sigma}\left(\boldsymbol{K}, i \omega_{m}\right) \equiv \Sigma_{\mu \nu, \sigma}\left(i \omega_{m}\right) \delta_{\mu \nu}$. Next, the lattice model (11) is mapped onto a set of effective quantum impurities subject to a dynamic bath $\mathcal{G}_{0, \sigma}\left(i \omega_{m}\right)$ that has to be determined self-consistently. Here we use a HirschFye quantum Monte Carlo (QMC) impurity solver; it not only provides access to finite temperatures but also has the advantage of simulating an effectively infinite bath.

In particular, for a given site-diagonal element of the bath Green's function $\mathcal{G}_{0, i \sigma}\left(i \omega_{m}\right)$, chosen to impose an initial stripe configuration, we use $L_{c}$ times the QMC solver to obtain the interacting Green's function $\mathcal{G}_{i \sigma}\left(i \omega_{m}\right)$ and then we extract through the local Dyson equation the corresponding self-energy, $\Sigma_{i \sigma}\left(i \omega_{m}\right)=$ $\mathcal{G}_{0, i \sigma}^{-1}\left(i \omega_{m}\right)-\mathcal{G}_{i \sigma}^{-1}\left(i \omega_{m}\right)$. Next, we compute a new bath Green's function using the self-consistency condition in a matrix form: ${ }^{\frac{9}{}} \mathcal{G}_{0, \sigma}^{-1}\left(i \omega_{m}\right)=\mathcal{G}_{\sigma}^{-1}\left(i \omega_{m}\right) \delta_{\mu \nu}+\boldsymbol{\Sigma}_{\sigma}\left(i \omega_{m}\right)$, with $\boldsymbol{G}_{\sigma}\left(i \omega_{m}\right)=\frac{1}{L_{u}} \sum_{\boldsymbol{K}} \frac{1}{\boldsymbol{G}_{0}^{-1}\left(\boldsymbol{K}, i \omega_{m}\right)-\boldsymbol{\Sigma}_{\sigma}\left(i \omega_{m}\right)}$. It couples all the impurity sites and hence the actual charge density at a given site depends effectively on the charge distribution at other sites in the stripe phase. The procedure is repeated till convergence is reached. Finally, we represent the lattice Green's function, $\boldsymbol{G}_{\sigma}^{-1}\left(\boldsymbol{K}, i \omega_{m}\right)=$ 

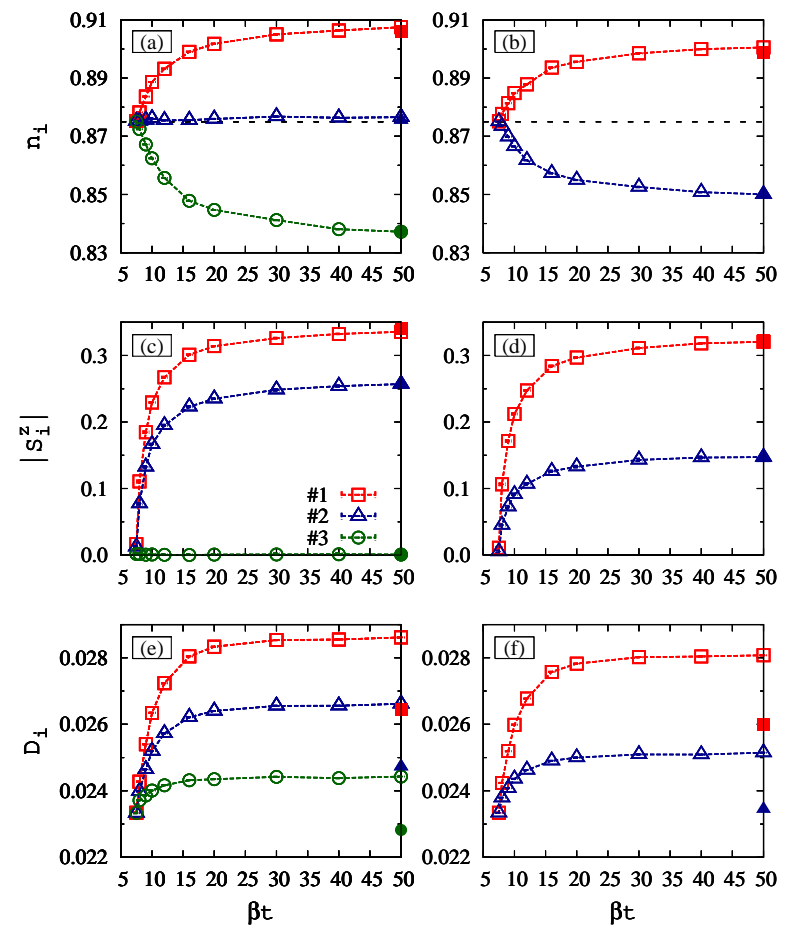

FIG. 1: (Color online) Temperature dependence of the: $(a, b)$ local charge density $n_{\mathrm{i}}$, (c,d) magnetization $\left|S_{\mathrm{i}}^{z}\right|$, as well as (e,f) double occupancies $D_{\mathrm{i}}$ at the inequivalent sites in the $\mathrm{SC}$ (left) and $\mathrm{BC}$ (right) stripe phase at doping $x=1 / 8$. Filled symbols at $\beta t=50$ were obtained with $t^{\prime}=-t / 3$.

$\boldsymbol{G}_{0}^{-1}\left(\boldsymbol{K}, i \omega_{m}\right)-\boldsymbol{\Sigma}_{\sigma}\left(i \omega_{m}\right)$, in the original BZ as follows, $g_{\sigma}\left(\boldsymbol{k}, i \omega_{m}\right)=\frac{1}{L_{c}} \sum_{\mu, \nu=1}^{L_{c}} e^{i \boldsymbol{k}\left(\boldsymbol{a}_{\mu}-\boldsymbol{a}_{\nu}\right)} \boldsymbol{G}_{\sigma}\left(\boldsymbol{K}, i \omega_{m}\right)$, and by rotation to the real-frequency axis via stochastic analytical continuation method, 10 extract the $\boldsymbol{k}$-resolved singleparticle spectral function $A(\boldsymbol{k}, \omega)=-\frac{1}{\pi} \operatorname{Im} \sum_{\sigma} g_{\sigma}(\boldsymbol{k}, \omega)$.

We begin by showing in Fig. 1 1 the temperature evolution of the local charge $n_{\mathrm{i}}=\sum_{\sigma}\left\langle c_{i \sigma}^{\dagger} c_{i \sigma}\right\rangle$ and magnetization $\left|S_{\mathrm{i}}^{z}\right|=\frac{1}{2}\left|\left\langle n_{i \uparrow}-n_{i \downarrow}\right\rangle\right|$ densities as well as changes in double occupancies $D_{\mathrm{i}}=\left\langle n_{i \uparrow} n_{i \downarrow}\right\rangle$ in the two possible vertical stripe patterns at doping $x=1 / 8$ : (i) site-centered (SC) one with enhanced hole density at the nonmagnetic domain walls (DW) that separate AF spin domains and (ii) bond-centered (BC) one consisting of hole-rich ladders with a weak ferromagnetic order on the rungs separating AF ladders. In fact, using the symmetry of the self-energy in the spin sector, it is possible to reduce the number of the impurity sites that must be solved down to 3 (2) sites in the SC (BC) stripe phase, respectively, in a magnetic stripe unit cell with $L_{c}=8$ atoms. Guided by the experimental data one can expect a complex process of melting of the stripe order, possibly via an intervening phase with a pure charge order $\underline{\underline{6}}$ We find instead that the spin and charge orders disappear simultaneously at $\beta t \simeq 7$ and the overall melting is reminiscent of disappearance of the mean-field stripe phase in the overdoped regime $\stackrel{11}{1}$ This follows from Fig. 1 where one observes a
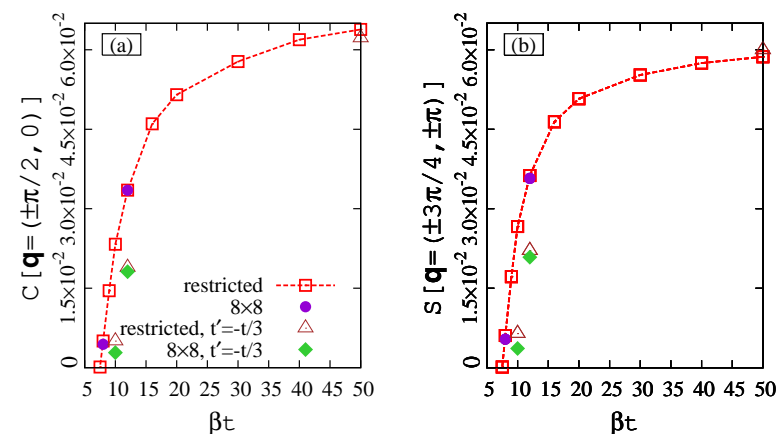

FIG. 2: (Color online) Intensity of the: (a) charge $C(\boldsymbol{q})$ and (b) spin $S(\boldsymbol{q})$ structure factor maxima at the wave vectors corresponding to the SC stripe phase with the charge (spin) unit cell containing 4 (8) atoms, respectively. For clarity, $C(\boldsymbol{q})$ was multiplied by a factor of 4 .

simultaneous increase in the charge $n_{\mathrm{i}}$ and spin $S_{\mathrm{i}}^{z}$ order parameters on decreasing $T: \frac{12,13}{2} \mathrm{~A}$ possible source of discrepancy between the numerical and experimental data lies in the absence of spatial fluctuations inherent to the RDMFT approach. Indeed, the physics of the proximity to phase separation in the cuprates is not correctly captured since the later instability corresponds to a long-wavelength instability. Alternatively, the melting of the stripe order in the layered cuprates might well be controlled by the interplane coupling allowing for a phase transition to occur at a finite-temperature. Indeed, it is not evident that the inter-plane coupling affects the charge and spin orders in the same way. We also note that the enhancement of the double occupancies in the low $T$-regime gives insight into the mechanism of the stripe formation. The kinetic energy gain acts as a driving force behind the stability of charge stripes along which doped holes can propagate coherently as seen in the corresponding spectral functions (see later).

To test the robustness of the stripe phase and exclude possible stripe meandering into the AF domains we release all the constraints and solve self-consistently an $8 \times 8$ stripe supercell at $\beta t=8$ and 12 where the use of the QMC solver albeit time expensive is nevertheless feasible. Further, in order to facilitate the analysis, it is convenient to compare the intensity of the cusps in the charge $C(\boldsymbol{q})=\frac{1}{N} \sum_{\boldsymbol{r}} e^{\boldsymbol{q} \cdot \boldsymbol{r}}\left\langle n_{0}\right\rangle\left\langle n_{\boldsymbol{r}}\right\rangle$ and spin $S(\boldsymbol{q})=$ $\frac{1}{N} \sum_{r} e^{\boldsymbol{q} \cdot \boldsymbol{r}}\left\langle S_{0}^{z}\right\rangle\left\langle S_{\boldsymbol{r}}^{z}\right\rangle$ structure factors at the appropriate wave vectors $\boldsymbol{q}_{c}=( \pm \pi / 2,0)$ and $\boldsymbol{q}_{s}=( \pm 3 \pi / 4, \pm \pi)$. Again, as shown in Fig. 2 we find that the temperature dependence of both structure factors is very similar. Therefore, we conclude that relaxing the constraint has no overall effect even in the vicinity of the phase transition where thermal fluctuations are important.

The signature of the stripe order emerges predominantly in the low-energy features of the single-particle spectral function $A(\boldsymbol{k}, \omega)$. Figs. B(a-c) and 4 plot this quantity as a function of temperature. Let us first concentrate on the nodal direction of the BZ. Here, we note 


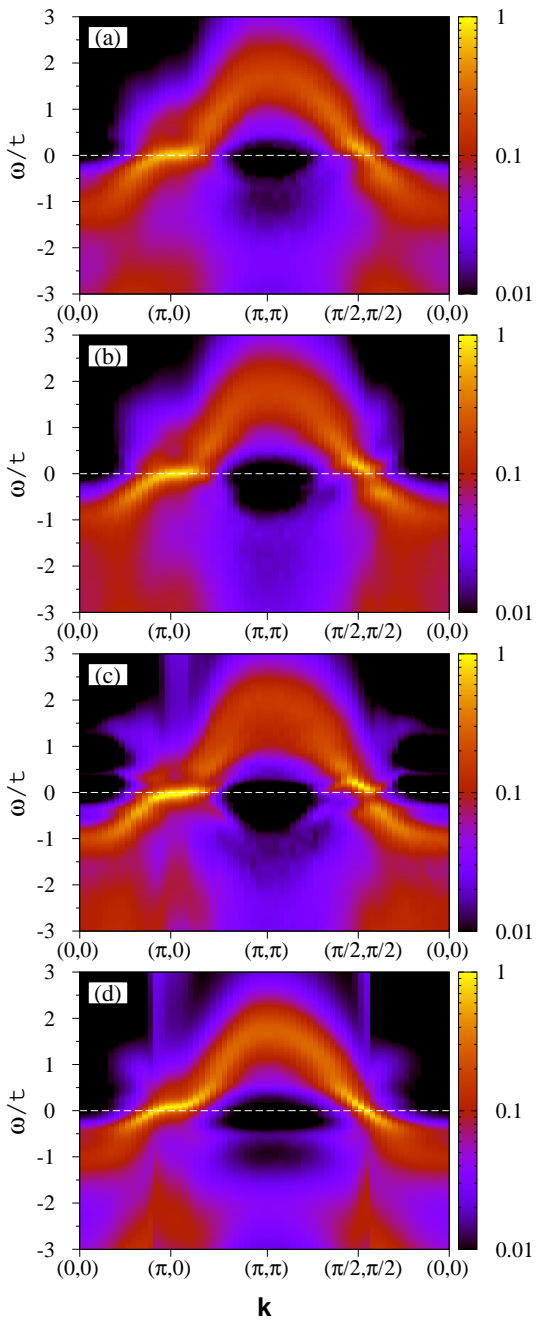

FIG. 3: (Color online) Evolution of the low-energy part of the single-particle spectral function $A(k, \omega)$ in the SC stripe phase: (a) $\beta t=12$, (b) $\beta t=16$, and (c) $\beta t=50$; the SC and $\mathrm{BC}$ stripe phases are dynamically indistinguishable. Panel (d) shows $A(\boldsymbol{k}, \omega)$ in the PM phase at $\beta t=50$.

that thermally disordered stripes below $\beta t=12$ (albeit clearly seen in the order parameters in Fig. (1) leave $A(\boldsymbol{k}, \omega)$ in the nodal direction almost intact. These findings are consistent with Ref. 14 suggesting that the nodal $A(\boldsymbol{k}, \omega)$ in the stripe system may arise in the presence of disorder or due to fluctuations of stripes facilitating penetration of the $\mathrm{AF}$ domains by holes. In contrast, a further reduction of temperature strengthens stripe order and modifies its spectral properties. Indeed, already at $\beta t=16$ one finds along the nodal direction two separated features originating from the single quasiparticle (QP) peak. It results in the depletion of $A(\boldsymbol{k}, \omega)$ at the Fermi level $\varepsilon_{F}$. Consequently, as depicted in Fig. 5(a), the QP peak seen at $\varepsilon_{F}$ at $\beta t=8$ in the density of states $N(\omega)$ is superseded by a pseudogap that opens up at $\beta t=16$ just below $\varepsilon_{F}$. Moreover, when moving towards the $S=(\pi / 2, \pi / 2)$ point in Fig. 团 spectral weight
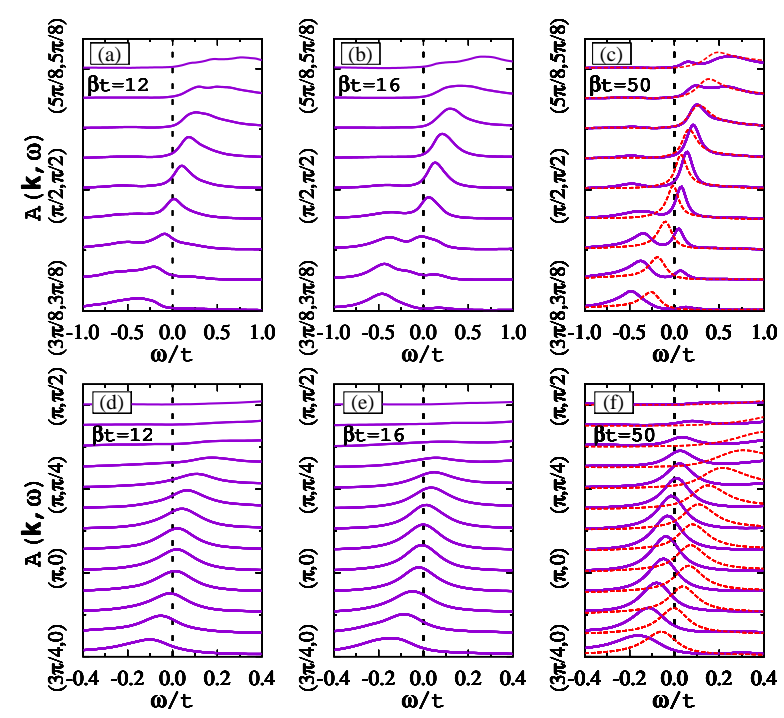

FIG. 4: (Color online) Temperature dependence of the lowenergy part of the spectral function $A(\boldsymbol{k}, \omega)$ in the SC stripe phase in the vicinity of: (a-c) the nodal $S=(\pi / 2, \pi / 2)$ and (d-f) antinodal $X=(\pi, 0)$ points. For comparison, dashed line in panels (c) and (f) shows $A(\boldsymbol{k}, \omega)$ in the PM phase.

is transferred gradually from the lower to the upper QP peak whose maximum is far above $\varepsilon_{F}$ at the $S$ point. Hence, in agreement with the previous $T=0$ RDMFT studies of the stripe order predicting opening a gap for charge excitations along the diagonal direction,,$\underline{\underline{7}}$ it seems that at the RDMFT level, well developed metallic stripes are incompatible with the observed in $\mathrm{La}_{2-x} \mathrm{Sr}_{x} \mathrm{CuO}_{4}$ (LSCO) and LNSCO nodal quasiparticles $\frac{15}{2}$ Altogether, noting that the measured nodal $A(\boldsymbol{k}, \omega)$ is more intense in LSCO (possible dynamic stripes) than in LNSCO (static stripes) and comparing with the temperature dependence of $A(\boldsymbol{k}, \omega)$ shown in Fig. 团 we confirm that the intensity of the nodal $A(\boldsymbol{k}, \omega)$ provides a measure of the stripe disorder.

Similarly to the case of the nodal direction, it is only above $\beta t=12$ that we observe the formation of a flat band near the antinodal $X=(\pi, 0)$ point located just below $\varepsilon_{F}$. This flat band can originate from the renormalization of the effective mass due to the frequency dependence of the self-energy - as observed in the DMFT studies of models of heavy-fermions $\underline{16}$ - or from its spatial dependence. To address this issue, we calculate $A(\boldsymbol{k}, \omega)$ in the uniform paramagnetic (PM) phase at the same doping $x=1 / 8$. As depicted in Figs. 3(d) and 4, neither the flat QP band at the $X$ point develops nor the gap around the $S$ point opens up in this case. Moreover, the Matsubara axis mass-renormalization factor i.e., derivative of the imaginary part of the self-energy taken at the DW w.r.t. the smallest Matsubara frequency $\left.\frac{-\partial \operatorname{Im} \Sigma_{\mathrm{DW}}\left(i \omega_{m}\right)}{\partial \omega_{m}}\right|_{\omega_{m}=\pi T}$ does not change across the stripe energy scale $\beta t \simeq 16$ [see Fig. [5(b)] at which visible changes in $A(\boldsymbol{k}, \omega)$ develop. Hence, it is the site-dependence of the self-energy 

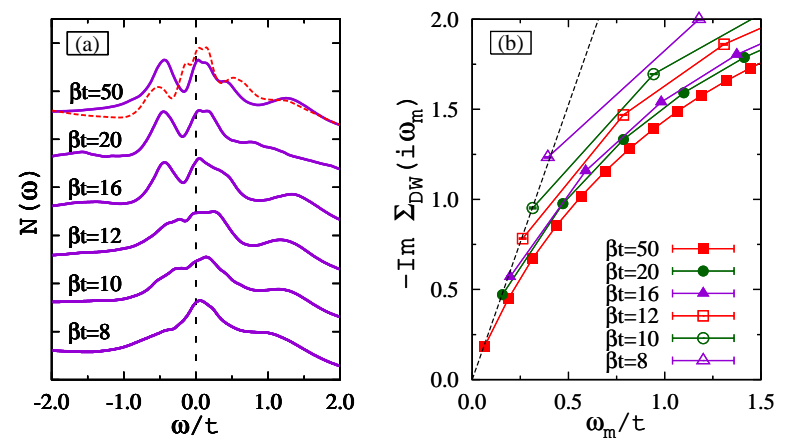

FIG. 5: (Color online) (a) Temperature dependence of the density of states $N(\omega)$ in the SC stripe phase and (b) imaginary part of the self-energy at the DW. Dashed line in panel (a) shows $N(\omega)$ in the PM phase while in panel (b) depicts the result of a linear fitting of data points at the smallest Matsubara frequency $\omega_{m}=\pi T$.

which is responsible for the substantial differences in the angle-resolved and integrated spectral functions between the PM and stripe phases in the low- $T$ limit.

Finally, let us briefly address the influence of the nextnearest-neighbor hopping $t^{\prime}$ on the melting of the stripe order. On the one hand, Fig. [1]shows that the charge and spin profiles at our lowest temperature $\beta t=50$ are only weekly affected by a finite $t^{\prime}=-t / 3$. On the other hand, a destabilizing function of $t^{\prime}$ is seen as a strong reduction of $D_{i}$ which signalizes reduced kinetic energy gain. The stripe order becomes more fragile and vanishes already at $\beta t \simeq 9$. While we have again not found a spontaneous tendency towards stripe meandering in the $8 \times 8$ unit cell, one nevertheless observes enhanced discrepancies w.r.t. the $t^{\prime}=0$ case indicating the frustrating role of $t^{\prime}$.
In summary, our RDMFT calculations reveal a strong temperature dependence of $A(\boldsymbol{k}, \omega)$ in the stripe phase. The dominant contribution to the electron mass renormalization at the antinodal points originates from the spatial dependence of the self-energy. Furthermore, it is only well below the transition temperature that a charge gap opens in the nodal direction. The melting of the stripe phase is mean-field-like since both charge and spin modulations vanish simultaneously. Within the RDMFT this was shown to be a robust result with respect to both $t^{\prime}$ and the size of the stripe supercell. The discrepancies with the experimental situation in LESCO, in which a two-step melting is observed, can be understood in terms of the absence of spatial fluctuations inherent to the RDMFT approach. Additional argument comes from the recent dynamic cluster approximation studies showing that the short-range AF correlations in the PM phase are sufficient for the occurrence of phase separation $\underline{17}$ Hence, it would be interesting to investigate whether the tendency towards phase separation can develop into a pure charge stripe order seen in LESCO. While other terms such as the long-range Coulomb interaction and interplane coupling might also be necessary, a systematic improvement of the RDMFT aimed at capturing spatial fluctuations is presently under progress.

\section{Acknowledgments}

We thank the LRZ-Münich and the Jülich Supercomputing center for a generous allocation of CPU time. M.R. acknowledges support from the Alexander von Humboldt Foundation. F.F.A. thanks the DFG for financial support.
1 M. Vojta, Adv. Phys. 58, 699 (2009).

2 C. Castellani, C. Di Castro, and M. Grilli, Phys. Rev. Lett. 75, 4650 (1995); O. Zachar, S. A. Kivelson, and V. J. Emery, Phys. Rev. B 57, 1422 (1998).

${ }^{3}$ C. N. A. van Duin and J. Zaanen, Phys. Rev. Lett. 80, 1513 (1998).

4 N. Ichikawa et al., Phys. Rev. Lett. 85, 1738 (2000).

5 J. M. Tranquada et al., Phys. Rev. B 78, 174529 (2008).

6 J. Fink et al., Phys. Rev. B 79, 100502 (2009).

7 M. Fleck, A. I. Lichtenstein, E. Pavarini, and A. M. Oleś, Phys. Rev. Lett. 84, 4962 (2000); M. Fleck, A. I. Lichtenstein, and A. M. Oleś, Phys. Rev. B 64, 134528 (2001).

8 A. Georges, G. Kotliar, W. Krauth, and M. J. Rozenberg, Rev. Mod. Phys. 68, 13 (1996); T. Maier, M. Jarrell, T. Pruschke, and M. H. Hettler, ibid. 77, 1027 (2005).

${ }^{9}$ Note that the Kronecker delta $\delta_{\mu \nu}$ is necessary to relate the diagonal elements of the Green's function $\mathcal{G}_{\sigma}\left(i \omega_{m}\right)$ to the local Green's function $\mathcal{G}_{i \sigma}\left(i \omega_{m}\right)$.

${ }^{10}$ K. S. D. Beach, arXiv:cond-mat/0403055 (2004).
11 M. Raczkowski, R. Frésard, and A. M. Oleś, Physica B 359-361, 780 (2005).

12 José A. Riera, Phys. Rev. B 64, 104520 (2001); ibid. 65, 064524 (2002).

13 Y. Shibata, T. Tohyama, and S. Maekawa, Phys. Rev. B 64, 054519 (2001); T. Tohyama, S. Maekawa, and P. Prelovšek, ibid. 67, 180502 (2003).

14 M. I. Salkola, V. J. Emery, and S. A. Kivelson, Phys. Rev. Lett. 77, 155 (1996); M. Granath, V. Oganesyan, D. Orgad, and S. A. Kivelson, Phys. Rev. B 65, 184501 (2002).

15 X. J. Zhou et al., Phys. Rev. Lett. 86, 5578 (2001).

16 M. Raczkowski, P. Zhang, F. F. Assaad, T. Pruschke, and M. Jarrell, Phys. Rev. B 81, 054444 (2010).

17 A. Macridin, M. Jarrell, and T. Maier, Phys. Rev. B 74, 085104 (2006); E. Khatami, K. Mikelsons, D. Galanakis, A. Macridin, J. Moreno, R. T. Scalettar, and M. Jarrell, ibid. 81, 201101 (2010). 